\title{
Embracing Safe Ground Test Facility Operations and Maintenance
}

\author{
Steven C. Dunn ${ }^{1}$ \\ Jacobs Technology Inc., NASA Langley Research Center, Hampton, VA, 23681 \\ Donald R. Green ${ }^{2}$ \\ Jacobs Technology Inc., NASA Langley Research Center, Hampton, VA, 23681
}

\begin{abstract}
Conducting integrated operations and maintenance in wind tunnel ground test facilities requires a balance of meeting due dates, efficient operation, responsiveness to the test customer, data quality, effective maintenance (relating to readiness and reliability), and personnel and facility safety. Safety is non-negotiable, so the balance must be an "and" with other requirements and needs. Pressure to deliver services faster at increasing levels of quality in under-maintained facilities is typical. A challenge for management is to balance the "need for speed" with safety and quality. It's especially important to communicate this balance across the organization - workers, with a desire to perform, can be tempted to cut corners on defined processes to increase speed. Having a lean staff can extend the time required for pre-test preparations, so providing a safe work environment for facility personnel and providing good stewardship for expensive National capabilities can be put at risk by one well-intending person using at-risk behavior. This paper documents a specific, though typical, operational environment and cites management and worker safety initiatives and tools used to provide a safe work environment. Results are presented and clearly show that the work environment is a relatively safe one, though still not good enough to keep from preventing injury. So, the journey to a zero injury work environment - both in measured reality and in the minds of each employee - continues. The intent of this paper is to provide a benchmark for others with operational environments and stimulate additional sharing and discussion on having and keeping a safe work environment.
\end{abstract}

\section{Introduction}

C ONDUCTING integrated operations and maintenance in wind tunnel ground test facilities requires a balance of meeting due dates, efficient operation, responsiveness to the test customer, data quality, effective maintenance (relating to readiness and reliability), and personnel and facility safety. Safety is non-negotiable, so the balance must be an "and" with other requirements and needs. Pressure to deliver services faster at increasing levels of quality in under-maintained facilities is typical.

A challenge for management is to integrate the "need for speed" with safety and quality. It's especially important to communicate this balance across the organization - workers, with a desire to perform, can be tempted to cut corners on defined processes to increase speed. Having a lean staff can extend the time required for pre-test preparations, including hazard analyses (and hazard identification), so providing a safe work environment for facility personnel and providing good stewardship for expensive National capabilities can be put at risk by one wellintending person using at-risk behavior to accomplish what he or she thinks is the most important work. Pressure to achieve operations or maintenance to meet pre-defined due dates, often exacerbated by added or changing work requirements from the test customer, can be tremendous. It is the responsibility of everyone involved in the work, with facility principles leading, to ensure accomplishment of planned work and any process deviations are accomplished safely. This is a challenge that must, and can, be met.

${ }^{1}$ Chief Engineer, ROME Group, NASA Langley Research Center, Mail Stop 267, Hampton, VA, 23681, and AIAA Senior Member.

${ }^{2}$ Safety Manager, ROME Group, NASA Langley Research Center, Mail Stop 440, Hampton, VA, 23681, and AIAA Senior Member. 
This paper documents a specific, though typical operational environment and cites management and worker safety initiatives and tools used to provide a safe work environment. Results are presented and clearly show that the work environment is a relatively safe one, though still not good enough to keep from preventing injury. So, the journey to a zero injury work environment - both in measured reality and in the minds of each employee continues. It is hoped that the sharing of these lessons and processes may help others to springboard their organizations toward the norm that everyday, everyone goes home unhurt.

\section{Safety Performance Requirements and Expectations}

Over the last century, the tolerance and expectations regarding workplace safety have changed dramatically. The attitude that accidents are "just going to happen" and the general acceptance of some number of injuries has turned into a requirement that there be zero injuries and everyone goes home unhurt at night. Driven initially by government requirements and economics, high performing companies now realize that a safe work environment drives performance gains through improved work planning, engaged workers, less interruptions, continued availability of skilled people, and a culture of caring. Many owners/customers share the understanding that a safe work environment is good for all and often make safety a major criterion of selection of a company to do work. NASA is such a customer, with safety performance requirements well exceeding those of the Occupational and Safety Health Administration (OSHA). Over the last one hundred years, it has become apparent to most that the cost of poor safety performance - in terms of injury cost (including family impacts and loss of a valued worker), equipment damage, and sometimes lost production - well exceeds any gains from using unsafe practices in the name of efficiency.

Consider an example of the cost of poor safety: As a manager of a small electrical construction company, you often and bid on and win work in the city as a subcontractor for larger companies. The jobs usually involve accomplishing some specific work. You employ about 100 people, mostly electricians and apprentices/helpers. You consider your safety record to be really good - your injury rate was actually slightly below your industry average last year. You recently won a job at ACME Production Plant to install a new breaker box in an existing system. Your team went to the job site late in the afternoon yesterday to review the job and to work with the ACME workforce to accomplish system lock out and tag out. This morning, two of your best electricians and a helper went to the work site to do the job, which was expected to take all day to complete. The schedule was fairly aggressive, but it had been done before and the electricians were due on a different job tomorrow.

Upon arrival the team got to work. The team lead, a senior electrician with 20+ years experience recommended that they just get to work since they locked everything out yesterday. When asked about verifying it today, he commented that it would just be a waste of precious time and on every other job he did verification it was still de-energized. So, after donning the appropriate personal protective equipment (PPE), the lead electrician went to the old breaker box and cut the electrical supply line. Upon cutting into the line, there was a huge flash and explosion. The electrician, despite his PPE, was burned badly on his hands and arms and he was thrown back against a wall. The other two workers were also injured, though not as severely. Somehow (does it really matter how?) the system had been reenergized on second or third shift.

What did this ten minute shortcut of skipping system verification cost?

To the lead electrician: He's still alive (thanks to the PPE), but badly burned. He faces a year or more of painful dead skin stripping, skin grafts, and then rehabilitation. He won't be able to use his hands for anything for several months. His family will be under great stress emotionally and financially (since he is the primary income source for the family, plus additional health related costs). He'll hopefully have full function in his hands after all this, but the doctors don't know for sure.

To the other electrician and helper: They experienced lesser burns and flash burns to their eyes. They'll be very uncomfortable and out of work for at least a couple of weeks, but are expected to recover.

To ACME: Their work site was shut down, stopping production for a week. They fired you for the safety violation of not verifying the LOTO. Now they have to hire another contractor to do the job. They also are suing you to recover their costs for damage the surge caused to some electrical equipment.

To you and your company: You have direct costs from covering any injury-related costs that insurance didn't cover. Your company has been fired by ACME and is being sued. You and your company are being investigated by OSHA and likely will incur a significant fine - hopefully you as manager won't be criminally charged. Your company's reputation has been trashed and will make it difficult to capture new work. You've got two of your best people out, one for longterm. But that may work out; you likely will be laying off people since new work is limited.

To summarize, ten minutes for three people was saved. That's a total of 30 minutes pay and 10 minutes clock time. Was it worth it?

\section{A. OSHA and Safety}

The US Occupational Safety and Health Administration (OSHA) provides both direction and guidance on ensuring a safe workplace. Direction is provided through a multitude of regulations which are found in Title 29 of 
the Code of Federal Regulations. Guidance, both to help provide a safe workplace and navigate through the regulatory minefield, is provided through the Voluntary Protection Program.

The OSHA Voluntary Protection Program (VPP) sets performance-based criteria for a company's safety and health program and then assesses those programs using an application review and onsite evaluation by a team of OSHA and industry safety and health experts. Based on the review and evaluation, successful applicant organizations are rated either Merit or Star. Regardless of rating, the average VPP worksite has a Days Away Restricted or Transferred (DART) case rate approximately half of the average for comparable organizations doing similar work. ${ }^{1}$

In the case of Jacobs Technology. Inc. (JT) .

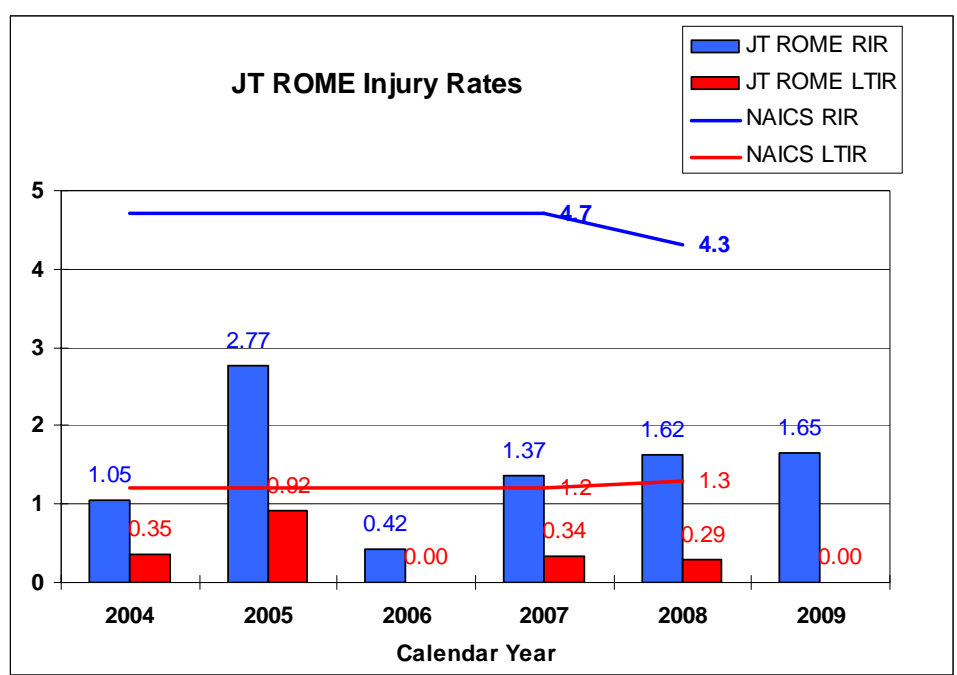

Figure 1. Incidence rates - detailed industry level - $2008^{3}$

Injuries is 4.3, and the DART rate is 1.8. The ROME group's actual Recordable Injury rate for 2009 was 1.65 (38\% of the industry's average), and the DART rate was 0.0 - an indication of low injury severities (Figure 1). The ROME Group received its VPP Star rating in April, 2008.

\section{B. The Impact of Incidents}

For any program, a significant unplanned event (the traditional definition of an incident) has ramifications to the primary performance measurements of the program: cost, schedule and performance/quality. If someone is hurt, the impact goes far beyond there. The team member injured, co-workers, family, friends - all are affected much more than what is shown on the ledger sheet; but first, the impact to the program.

Cost: There may be direct costs to repair damage to a facility, base infrastructure, or test hardware. System and/or facility downtime resulting either from equipment damage or time required to investigate and understand the root causes (and, possibly, to take actions to reduce risk before proceeding) is lost revenue for the facility and results in a cost for staff that can't be productively utilized on other work. Schedule delays to the test program can have enormous cost effect on product development and at the least increases cost on the particular test project. Having an unhappy customer can also result in the loss of future business.

Schedule: Customers expect products to be delivered on time. Delays in testing can cause day-for-day slips in the product's delivery schedule. A significant incident can cause downtime of a facility for an extended period, driving a customer to a likely suboptimal facility and increasing product risk. Downtime may also be experienced if a wait is required to find and secure funding to repair damage. Long delays can also result in the loss of long-term business; planned customers make other plans and find other suppliers.

Performance/Quality: Customers require a product at a defined level of quality. It is the repeated experience of the authors that, for wind tunnel testing, data quality is the highest priority of the customer. Accidents can cause capability limitations (using what capabilities and capacities that are available), driving a trade-off between quality and schedule. Loss of a critical person, even temporarily, can impact facility performance and results.

Early in the ROME contract, NASA management provided a clear statement on their performance expectations. ROME, as operators of wind tunnel ground test facilities, needed to please Langley Research Center's (LaRC) customers while showing excellent stewardship of LaRC's facilities. Clearly, incidents in any form in the facilities are at odds with this operations mission expectation.

\section{Incident Financial Reports}

Based on data from the National Council on Compensation Insurance, Inc. (NCCI), even the relatively low injury rate experienced by JT ROME would have an estimated cost of over \$400,000 in both direct and indirect costs in $2009^{4}$. Since ROME is self-insured (backed by JT), these costs come directly out of profit; based on an organization's profit margin, the cost of an incident can require a significant amount of new sales to recoup.

Injured employees often fare even worse. Depending on the state in which the injury occurred, worker's compensation payments are a fraction of base pay. In Virginia, for example, an injured employee who is unable to 
return to work for a period longer than 1 week will receive $2 / 3$ of normal pay, up to a maximum of $\$ 885$ per week. ${ }^{5}$ For most of the skilled workforce at LaRC, this is a significant reduction in earnings.

Equipment repair/replacement costs must be considered as well. Many test articles and test fixtures are 'one-ofa-kind' assets, and may be irreplaceable. If repairs are fiscally feasible, they can drive the cost of a program to unacceptable levels, leading to reduced test scope or cancellation.

\section{Operational Environment}

This paper focuses on activities occurring in wind tunnel facilities at LaRC, including all phases of wind tunnel operation, maintenance, investment projects (maintenance and improvements), training, tours, and changes in staffing and responsibilities. The primary responsibility of the people in the facilities is to produce high quality test data and associated excellent customer service, while providing stewardship for facility people and equipment. Prior to the contract start, the organization consisted of nine contracts/contractors and multiple NASA organizations, driving extensive sequential operations. A significant tenant of the ROME contract was to combine the contractor organizations into one and work cohesively with NASA people at each facility on Day 1 . Tactical responsibilities would be transitioned from NASA to the contractor over an aggressive four year period, ending in essentially a turnkey tactical operation at each of five major wind tunnels. Transition would be accomplished while other activities, including test operations, continued.

A major contractual consideration and expectation by NASA was that the contractor would deliver significantly improved safety, immediately and throughout the contract. The existing overall safety record at Langley at contract start already was good, much better than industry averages as witnessed by LaRC becoming the first federal VPP Star site in 1998. NASA management wanted even better. Since the workforces in the facilities were combined contractor and civil servant, the responsibility to provide a safe workplace was jointly held, though the contractor could implement new methods immediately. Performance expectations coupled with a strong desire to deliver quality services even while the organization was building resulted in a number of safety challenges, the major challenges faced are shown in Table 1 , with the larger " $X$ " indicating greater areas of concern. The concerns related to different aspects of an organization and how they needed to be addressed: organization culture, safety program tools, and work processes. Table 1 also includes a cross-match of challenges to the requirements of conducting tests while integrating operations and maintenance and accomplishing a workforce transition. Thus, the creation of a safe work environment, even while improving productivity and quality, must account for these different elements - just implementing a program of mandated safety tools would necessarily fall short.

\section{A. The Role of Safety in an Operational Environment}

The expectation and goal of JT ROME management began and remains zero injuries across the contract, including subcontractors. An additional expectation is that, through partnering, injuries to civil servants can also be driven to zero. A significant effort has been expended (and continues) to work with every person that makes up the ROME contract to get them to truly believe that every person can and will go home every day without being injured. Safety is not just a program, a management initiative du jour, or even a priority. Safety is a core value, integrated in every activity. And when it becomes a core value, then people also take it home with them - really, it becomes a part of who they are.

There are inherent hazards in any activity. Identifying, understanding, and managing the risk associated with each hazard is the key to safe outcomes. Risk is often defined as a combination of probability that the hazard will occur,

\begin{tabular}{|c|c|c|c|}
\hline \multirow{2}{*}{ Challenges to Address } & \multicolumn{3}{|c|}{ Approach } \\
\hline & Cultural & Safety Tool & Process \\
\hline \multicolumn{4}{|l|}{ Test Projects } \\
\hline \multirow{6}{*}{$\begin{array}{l}\text { New models and equipment } \\
\text { Customer information often late } \\
\text { Aggressive schedules } \\
\text { Customer or NASA changes in plan } \\
\text { Unplanned downtimes/outages } \\
\text { Productivity requirements/expectations } \\
\end{array}$} & & $\mathbf{X}$ & \\
\hline & & & $\mathbf{x}$ \\
\hline & $\mathbf{x}$ & $\mathbf{X}$ & \\
\hline & & & $\mathbf{x}$ \\
\hline & & $\mathbf{X}$ & \\
\hline & & $\mathbf{X}$ & \\
\hline \multicolumn{4}{|l|}{ Integrating operations and maintenance } \\
\hline \multirow{5}{*}{$\begin{array}{l}\text { Pressure to hit due dates on planned downti } \\
\text { Overly optimistic investment project schedul } \\
\text { Existing conditions different than expected } \\
\text { Aging infrastructure with lower reliability: } \\
\text { Hurry to get it fixed/very reactive } \\
\text { Schedule pressure drives 'shortcuts' }\end{array}$} & & & $\mathbf{X}$ \\
\hline & & & $\mathbf{x}$ \\
\hline & & $\mathbf{x}$ & \\
\hline & $\mathbf{x}$ & & $\mathbf{x}$ \\
\hline & $x$ & $\mathbf{X}$ & $\mathbf{x}$ \\
\hline \multicolumn{4}{|l|}{ Workforce transition } \\
\hline \multirow{4}{*}{$\begin{array}{l}\text { Organizational cultural differences } \\
\text { Potentially adversarial relationships } \\
\text { Qualification of workforce (by skill, facility, technique) } \\
\text { Lean staff, long hours: }\end{array}$} & $\mathbf{X}$ & $\mathbf{x}$ & $\mathbf{x}$ \\
\hline & $\mathbf{X}$ & & $\mathbf{x}$ \\
\hline & & & $\mathbf{x}$ \\
\hline & $\mathbf{x}$ & $\mathbf{x}$ & \\
\hline \multirow{3}{*}{$\begin{array}{l}\text { Balance of efficiencies with reasonable work hours } \\
\text { One-deeps and subject matter experts overwork } \\
\text { Unplanned downtimes extend shifts } \\
\text { Desire to please test customers and NASA at any cost }\end{array}$} & $\mathbf{x}$ & $\mathbf{X}$ & \\
\hline & $\mathbf{x}$ & $\mathbf{x}$ & \\
\hline & $\mathbf{X}$ & & $\mathbf{X}$ \\
\hline
\end{tabular}

Table 1. Early contract safety challenges and related approach to address. 
measured against the likely severity if it does occur. The severity is often measured in terms of injury severity and/or amount of potential equipment damage. If the risk for a particular hazard is unacceptably high, some mitigation (activity modification, additional actions, etc.) must be implemented that will reduce activity risk to an acceptable level before proceeding. This process is formally present in most organizations. But how many people in those organizations think in terms of risk management when accomplishing their work? Probably many in organizations with excellent safety records!

There are a number of aspects that must be examined regarding the expectation that the workplace can and will be injury-free: how to achieve and sustain this culture and performance, effect on work performance, interactions with people and organizations outside of Langley, cost of implementation and sustainment, and individual expectations and buy-in (which must include the buy-in of their supervisors and managers).

\section{B. Is zero injuries an achievable goal?}

The authors believe a "zero injuries" level of performance can only be achieved through a designed process that overlays what Reason ${ }^{6}$ terms defense layers. In his "Swiss cheese model of system accidents", defensive layers can be engineered (alarms, physical barriers, automatic shutdowns, etc.), rely on people (surgeons, control room operators, etc.), and rely on procedures and administrative controls. The overlay of these defenses, when each defense is fully intact, is impermeable to the occurrence of an accident. But the reality is that each defense has holes - for instance, it is doubtful that any readers of this paper have yet to read a perfect procedure. And the holes, dependent on many variables (application, existing or changing conditions, performer competence, and many more), also change location and size over time. As Reason notes, "the presence of holes in any one 'slice' does not normally cause a bad outcome. Usually, this can happen when the holes in many layers momentarily line up to permit a trajectory of accident opportunity - bringing hazards into damaging contact with victims.”

The defense layer holes occur from two sources: active failures and latent conditions. Active failures are unsafe acts by people performing the work. Unsafe acts can be the result of incompetence (often driving incorrect responses to some impetus), well-intended shortcuts, or long-standing (but tolerated) bad practices. Latent conditions "are the inevitable 'resident pathogens' within the system"7. These conditions result from decisions made by defense layer designers and developers and can be either inherent defects or the result of some unintended situation or application (that then renders the defense insufficient). The latent condition may exist for a long time until the 'hole' aligns with other 'holes' and an accident results. A reasonable expectation is that 'holes' exist in every defensive layer, so the key is continuous improvement of designs and improved training of people combined with the positive active controls of the people.

Continuous improvement results from applying lessons learned, taking advantage of new developments (like new technologies), and listening to and incorporating improvement inputs from operators. This process strengthens (reduces 'holes') in existing defenses and can add additional defensive layers. It is also important to maintain already in-place defense layer capabilities - capability degradation can introduce new 'holes'.

Much of the safety-related literature, indeed, many corporate safety programs, focus on how to contain the effects of human error (or active failures). This is, obviously, appropriate. However, a high performing organization, one that aspires to zero injuries, must apply positive active controls from the people performing the work. This requires that the last defensive layer, the actions of the person or people performing the work, to be dynamic in terms of being constantly observant of weak signals that indicate either a changing or different condition than what was expected. It additionally requires that the observation be assessed in terms of hazard potential - getting this assessment right requires a fair degree of competence on the part of the worker. Lastly, the worker must be willing to stop the work, and the company culture and visible management leadership must support that action, until the potential hazard can be properly assessed and risk mitigating actions applied as necessary. Weick ${ }^{8}$ cites a study he conducted in which nuclear plant operators immediately readjusted dials and system commands when an automated system didn't respond as expected. Reason ${ }^{9}$, in a detailed study of a healthcare accident, argues that "organizational accident sequences could be thwarted at the last minute" by doctors and nurses on the front line if they had acquired "some degree of error wisdom". So people must be trained to be actively observant in scanning for process deviations. Since workers typically work together with others on a job, even with variability of background, if 'error wisdom' is resultant from training and certification processes, multiple positive active control layers can be added that will prevent an accident from happening.

Lastly, numerous examples can be found of organizations and projects that have achieved zero injuries over hundreds of thousands, even over a million hours work hours. Within Jacobs, a construction contract at Oak Ridge demonstrated safety performance of zero recordable injuries with over one million work hours through use of a multi-pronged safety culture and tools. So, yes, zero injuries is an achievable goal. 


\section{Does this level of safety affect work quality and performance?}

It is the observation of the authors that the prevailing culture in organizations in which an industry average number of people are getting hurt is that "accidents happen", are just something to deal with, and "I sure hope it doesn't happen to me." A corollary to this belief is that the introduction of a more intrusive safety program would take too much time and impede production. Various studies of organizational performance reject this notion and embrace that safety and quality work together in support of higher performance.

Mark Keroack ${ }^{10}$ led a study of academic medical centers to determine which demonstrated high performance on a broad-based composite measure of quality and safety attributes and found that high performing centers added service excellence. Focus of the high performers was on the patient (customer). Service, quality and safety were a source of competitive advantage in the higher performing medical centers. In a study of correlation between patient safety and hospital financial performance, two senior economists found that hospitals in financial trouble make more mistakes, concluding that patients face significantly greater odds of having adverse events from major surgeries in hospitals where profit margins have eroded. ${ }^{11}$ Zacharatos ${ }^{12}$ conducted two studies that each found organizations with high performance work systems experienced greater workplace safety, one in terms of fewer lost time injuries and the other in terms of fewer safety incidents.

It is the author's experience that effective work planning aligns with safer work practices and a better understanding of hazards associated with the work. In some cases, the processes used to identify hazards and assess risk improve work planning as well. For example, ROME employs a tool for each job known as a "Safe Plan of Action (SPA)". Planning forms, required for every job in the field, force the people getting ready to do the work to consider hazards they would likely face on the job. Then they develop mitigating actions for the hazards, including the use of safety equipment and appropriate tooling - really a form of job planning. This safety tool not only makes the front line folks aware of the potential hazards, but they will be better prepared to accomplish the work.

\section{Interactions with people and organizations outside of NASA Langley}

Subcontractors are utilized by ROME across the contract and have been a major challenge, consistently having higher injury rates than any other part of the ROME organization. Severity levels have also been higher. This lower performance is attributable to a difference in corporate cultures (and expectations), a push to meet schedules, a lack of understanding of hazards, and sometimes a pairing of unfamiliar people that causes communication gaps. The approach taken has been to partner with subcontractors, including:

- Meeting and discussing safety with senior management.

- Requiring every subcontractor person to attend safety training, provided by ROME, before beginning work on site.

- Increasing the presence of safety consultants/observers at the work site.

- Requiring greater presence and visibility of ROME construction managers.

- Requiring adoption of ROME tools, such as SPAs.

- Lack of tolerance for unsafe activities, especially for repetitive unsafe activities.

- Ensuring that anyone can stop work at any time if an unsafe condition is detected or suspected.

- As a measure of last resort, after repeated safety incidents, a company can be (and has been) barred from future work.

In the wind tunnels, ROME and NASA team to provide a safe working environment for test customers. Measures include:

- Coordination during pretest planning regarding equipment (including test models and support equipment) and personnel safety, including facility and test technique peculiar hazards

- Most facilities require all personnel to attend a facility safety briefing prior to starting work

- Personal protective equipment, if not already in hand, is provided as needed

- During testing, defined facility and test/model equipment limits are adhered to by operators

- Facility personnel, contractor and civil servant, watch out for each other and for visitors

Visitors to buildings and facilities also receive appropriate safety briefings or may be escorted by personnel trained and aware of hazards. Tour paths are designated and large groups include a tailing person to make sure no one gets off track. If hazards may be present on a tour path, either the hazard risk is mitigated or the tour path changed. If a visitor needs to enter an area requiring personal protective equipment, it is provided. 


\section{E. Is this cost prohibitive?}

A safety program and the establishment and nurturing of a caring culture drives a significant up-front cost to an organization. Costs include salaries of safety and environmental professionals, training, personal protective equipment, tool and equipment replacement and upgrades, performance incentives, "fresh-eye" observations with findings and recommendations, and potentially, facility upgrades (to address unsafe conditions). Tangible costs related to safety for a midsize organization (200-500 people) could range from $\$ 400,000$ to over a million dollars. If an organization is performing at an industry average of 4 injuries for every 200,000 hours worked, the average cost for a 500 person organization due to injuries alone is approximately $\$ 1.2$ million. ${ }^{13}$ For every person your program prevents from getting hurt, you save an average of $\$ 55,000$. You also prevent the trauma to your employee and his or her family and his or her workmates, the costs for investigating the accident, any production downtime, and you retain the services of your employee.

Fitch and Fitch ${ }^{14}$ reviewed case study literature regarding back injuries, citing that back injuries account for $40 \%$ of recorded absences from work and are the most costly medical condition in America. They also found that back injuries are generally repetitive trauma injuries - it has been estimated that only 4 percent of back injuries are the result of a single-incident injury. Their review and analysis indicated "back injury prevention programs can be cost effective in industry". Leisten ${ }^{15}$ cites reduced costs and downtime in the solid waste industry that can result from an effective safety program. Additionally, for a company with a tight budget, she suggests the approach of reviewing past losses to determine the most dangerous exposures, then developing tactics to predict and evade those hazards.

Atrinno ${ }^{16}$ documented how risk managers are finding it easier to justify the cost of installing fire-safety systems due to research and technology resulting in reduced insurance premiums and fewer property losses. Linda Johnson, the benefits administrator at Rosendin Electric, noted that half of their injuries were back or eye related. ${ }^{17}$ Back injuries cost the company between $\$ 15.000$ and $\$ 20,000$ each and due to their recurring nature, it was driving insurance premiums up and impacting their injury metrics, affecting their ability to gain new contracts. One simple measure they implemented was to issue, with training, back support belts - by saving just two employees from experiencing a back injury resulting in surgery, the program would pay for itself.

Thus, the cost of an affective safety program and supporting a caring culture can be significant. But the cost of neglecting safety often costs much more in direct costs, impact to the workforce individually and collectively, production downtime, possible litigation, equipment repair, and other prescribed accident investigation recommendations.

\section{F. How can individual buy-in and willing participation happen?}

Literature that speaks of organizational culture often states that culture change usually takes a fairly long time months to years. When the safety performance of an organization is poor, or even average, there isn't time to wait months or years for change to happen. There must be a combination of near-term and long-term measures put in place initially and then monitored real-time in response to changing conditions, weaknesses in the initial rollout, and ongoing performance. To achieve individual buy-in that zero injuries is achievable, it is imperative that all management personnel not only be bought in but must set the example for safe working behavior.

On the first day of the ROME contract (a Saturday) all managers and supervisors, many coming from the nine predecessor contracts, met for safety training and discussion. It was an indoctrination and a clear delineation of expectations related to safety processes and performance. This group was required to ramp up quickly in knowledge of safety tools, communicating expectations in a caring yet clear way, and getting out into the workplace to observe and mentor workers in safe, effective work practices. This process was integrated with understanding and improving work quality.

Many tools (discussed in Section IV of this paper) were implemented from the beginning of the contract. Meetings with personnel made clear that safety was non-negotiable and that use of these tools was a job requirement. Some people, with work efficiency in mind, objected to the time it took to accomplish some of the processes, but it was explained that a few small losses of time to plan and accomplish work safety would pay back many times over across a year. Implementing tools at the beginning was only a start. Ongoing engagement, initially by management at all levels, then by co-workers - especially subject matter experts - would be required to keep people engaged and really using the tools for the life of the contract (and hopefully beyond).

Probably the single biggest thing that gains buy-in of the safety program and culture is backing up what's preached. It is sometimes very inconvenient to stop work to assess a perceived unsafe condition and then, if required, to take some mitigating action. However, the organization must address safety concerns directly, with the supervisor backing up the worker that made the observation (and his or her management providing additional 
backup). In a strongly performing organization, most people don't want to stop production, but they must know and feel that it is OK to do so if something isn't right and creating a potentially unsafe condition. Another significant area of backup is mitigating or correcting observed unsafe conditions in the workplace. Workers are requested to scan their workplaces on a regular basis and identify any unsafe conditions - trip/crush/spear hazards, headknockers, environmental issues, etc. In older facilities, some conditions may have existed for a long time and people just lived with it. Or, an improvement that corrected the condition may have been on somebody's unfunded requirements list. Even if the condition is expensive to correct, some mitigation must be effected to reduce risk to acceptable levels (reducing accident probability or potential severity or both).

Employee-run safety councils are another excellent way both to engage the front line workers and to also gain some very creative and thoughtful inputs on improvement to the safety program and culture. These people are living it, typically much more intimately than their supervisor or manager, and they should be listened to. ROME incorporates departmental employee safety councils, typically led by a line worker with management participation; often the manager or supervisor gets the actions! Additional safety councils have been formed to work issues associated with safety culture and with the overall use of safety tools. Interaction from across the contract is achieved at a monthly executive safety council, with all senior management, designated safety professionals, department and specialized safety council chairs present, along with other interested parties (including, occasionally, NASA representatives). Issues are reviewed and actions given; actions range from process changes to recognition of good stuff to gathering more information about how a process is working. Again, the managers usually get the lion share of the actions.

\section{G. Obstacles / opportunities to improve}

At the start of the ROME contract, different safety cultures, philosophies and attitudes were present in the incumbent workforce. Many of the people had worked through multiple contracts for multiple companies, so building a trust in each other and gaining a trust that ROME management was serious about safety and was likely different than what they were used to was a challenge. Yet, bringing multiple experiences and cultures to bear on safety and work process improvement was an opportunity. Work rules and cultural differences existed between NASA and ROME, initially a challenge and ultimately an opportunity to effect improvement together. The initial ROME statement of work recommended an aggressive schedule of facility transition to contractor tactical operations. Combined with a high operational workload, accomplishing work safety with newly trained people was a challenge. ROME and NASA management, working together, aligned schedules with safe working capacity so schedule didn't drive people to take shortcuts. It is likely that every industrial organization has obstacles to safety and quality that, when studied and understood, can be turned into opportunities for improvement - ROME was no different. It is important to not let obstacles be an excuse to allow unsafe working conditions or work practices.

\section{H. Cultivating Partnerships}

When the ROME contract began, one of the expectations from the customer was that ROME would become a safety asset to the NASA Langley Research Center. Ambitious metrics were set to be attained, and extensive oversight set into place. Over time, ROME met and exceeded those expectations, and the processes put into place were emulated. Over time, a relationship far beyond the traditional client/provider developed, and mutually beneficial policies and processes have been developed. Improvements in the customer-owned Lockout/Tagout and high-voltage electrical processes by joint efforts are two of many programs which have benefitted.

\section{ROME Safety Awareness and Analysis Tools}

The expectation that the ROME contractor at NASA Langley will provide a safe work environment for everyone within its areas of influence percolates to the very top of Jacobs Technology, Inc. and Jacobs Engineering (JE). It is expected that every injury and accident can be prevented. Any and every injury is taken very seriously and scrutinized by ROME and JT senior management for understanding of cause, development of lessons learned, and sharing those lessons across the company. Jacobs brought a number of safety tools to the ROME contract that augmented the already-in-place tools required by NASA.

\section{A. Risk assessment}

Rome employs a risk assessment process on all engineering (mechanical, electrical, information technology, and civil/building construction) projects with values above $\$ 100,000$, prior to each wind tunnel test, and on maintenance jobs based on factors such as exposure to hazards (high work, confined spaces, chemicals, materials, energy sources, etc.), one-of-a-kind activities, excessive age of facilities/equipment, or other perceived risks. Additionally, each 
wind tunnel and research area at NASA Langley are required to have an in-place, up-to-date hazard risk assessment available at the facility (part of a grouping of facility information known as the facility resume). The process is documented in the NASA Management System and supplemented in the ROME (ISO Certified) Business System, and consists of a classical hazard identification process, assessment of each hazard in terms of likelihood of occurrence (probability) and potential severity if it occurs, mapping of the combination to identify the risk assessment code (RAC) and risk zone, developing mitigators for high risk hazards, and an iterative reassessment of hazards with mitigators in place until risk level for each hazard is acceptable to proceed (Figure 2). The initial assumptions and added mitigators become requirements for the effort and are tracked through the NASA/ROME systems engineering processes for the life cycle of the effort/project. In addition to the formal risk assessment process, embedded in safety training for each person are elements in hazard identification and risk assessment. The goal is for each person on each project to actively think about what they are doing in terms of hazards, likelihood of hazard occurrence, and severity of injury (to themselves and/or others) and/or equipment damage if it happened - an ongoing mental risk assessment. When risk is thought to be too high, work should stop until more formal assessment and potential implementation of mitigators can be employed to drive down risk. This process is especially important as operations and maintenance work is accomplished on existing facilities where the existing condition may be different (due to usage/aging or other cause) than expected when the work was planned. It is similarly important for projects within or interfacing with existing facilities and hardware due to unplanned or unknown interaction effects introduced by the new work.

\section{B. ROME Safety Processes}

1) Safe Plan of Action (SPA). This is a core element of the Jacobs safety program. The SPA is a core element of the Jacobs Health, Safety, and Environmental (HSE) Program. It is used to accomplish pre-task planning whenever an activity presents a risk of injury, illness, environmental, or property damage, and is required for all ROME employees and subcontractors

2) Hazard Assessment Safety Action Plan (HASAP). This is another Jacobs HSE core element, and is used by

\begin{tabular}{|c|c|c|c|c|c|c|c|}
\hline \multicolumn{4}{|c|}{ Severity } & \multicolumn{4}{|c|}{ Incident Likelihood or Probability } \\
\hline$\underline{\text { Performance }}$ & $\underline{\text { Schedule }}$ & Equipment & Personnel & $\underline{\text { Frequent }}$ & Likely & Possible & $\underline{\text { Unlikely }}$ \\
\hline $\begin{array}{l}\text { Total Mission } \\
\text { Unachievable }\end{array}$ & $\begin{array}{l}>12 \text { week } \\
\text { overrun }\end{array}$ & $>\$ 1 M$ & $\begin{array}{l}\text { Fatality or total } \\
\text { permanent } \\
\text { disability }\end{array}$ & RAC 1 & RAC 1 & RAC 1 & RAC 2 \\
\hline $\begin{array}{l}\text { Major Mission } \\
\text { Achievement } \\
\text { Compromise }\end{array}$ & $\begin{array}{l}\text { 6-12 week } \\
\text { overrun }\end{array}$ & $\$ 500 \mathrm{~K}$ to $\$ 1 \mathrm{M}$ & $\begin{array}{c}\text { Permanent partial } \\
\text { disability }\end{array}$ & RAC 1 & RAC 1 & RAC 1 & RAC 2 \\
\hline $\begin{array}{l}\text { Mission Performance } \\
\text { Degraded }\end{array}$ & $\begin{array}{l}\text { 3-6 week } \\
\text { overrun }\end{array}$ & $\$ 250 \mathrm{~K}$ to $\$ 500 \mathrm{~K}$ & Days away & RAC 1 & RAC 1 & RAC 1 & RAC 2 \\
\hline $\begin{array}{l}\text { Minor Mission } \\
\text { Performance } \\
\text { Compromise }\end{array}$ & $\begin{array}{l}\text { 1-3 week } \\
\text { overrun }\end{array}$ & $\$ 1 \mathrm{~K}$ to $\$ 250 \mathrm{~K}$ & $\begin{array}{c}\text { OSHA } \\
\text { Recordable }\end{array}$ & RAC 1 & RAC 1 & RAC 2 & RAC 3 \\
\hline $\begin{array}{l}\text { Negligible Mission } \\
\text { Performance } \\
\text { Compromise }\end{array}$ & $\begin{array}{l}<1 \text { week } \\
\text { overrun }\end{array}$ & $<\$ 1 \mathrm{~K}$ & First aid & RAC 2 & RAC 2 & RAC 2 & RAC 3 \\
\hline $\begin{array}{c}\text { No Mission Performance } \\
\text { Compromise } \\
\text { w/Adaptation }\end{array}$ & $\begin{array}{l}\text { No overrun with } \\
\text { adaptation }\end{array}$ & $\begin{array}{l}\text { No cost with } \\
\text { adaptation }\end{array}$ & $\begin{array}{l}\text { Discomfort and/or } \\
\text { Fatigue }\end{array}$ & RAC 2 & RAC 2 & RAC 3 & RAC 3 \\
\hline $\begin{array}{c}\text { No Mission Performance } \\
\text { Compromise }\end{array}$ & No overrun & Cosmetic only & Inconvenience & RAC 3 & RAC 3 & RAC 3 & RAC 3 \\
\hline
\end{tabular}

RAC 1: A major safety concern which requires resolution (reduction of the RAC from 1 downward to 2 or 3) before operations can be initiated or resumed

RAC 2: A significant safety concern with risk sufficient to require acceptance by appropriate operations/facility and site safety authorities

RAC 3: A safety concern with risk sufficient to require acceptance by appropriate site safety authority

Figure 2. JT ROME probability/severity decision matrix 
project management to predict the hazards associated with the project and to define the resource commitment to mitigate those hazards.

3) Working-Group Level Shift-Start Meetings: Every day prior to beginning assigned work activities, ROME personnel meet in a shop-level environment to discuss pertinent safety issues they anticipate encountering during the day such as weather, concurrent or unusual activities, and other safety-related items. This is often led by the workers themselves, includes lessons-learned from previous experiences as they relate to that day's assignments, and concludes with the creation of SPA outlines to be completed at the work site.

4) Morning Stretching Program: While not mandatory, all ROME personnel are encouraged to participate in morning stretches. These are warm-ups, not calisthenics, and allow personnel to loosen up before performing strenuous activities. Since its inception, ROME injuries due to sprains/strains have decreased over $50 \%$.

5) Safe Backing Program: ROME responded to an initial high vehicle backing incident rate by implementing a vehicle backing policy. Root cause analysis of the incidents consistently pointed to inadequate situational awareness. Discussion with ROME vehicle operators failed to obtain the desired results, so a policy of either using a spotter during backing or the driver walking around the vehicle was implemented. Since implementing this policy, vehicle backing incidents have decreased approximately $70 \%$.

6) Safety Observation Reports (SORs): The SOR is an important tool Jacobs uses across contracts for anyone to document safety observations in terms of condition and/or work practices. The SOR is used to document both potential safety issues as well as exemplary, positive safety practices. The observer describes what was observed and the associated hazard. If risk is deemed high, work is stopped until the hazard is better understood (to be lower risk) or the risk is mitigated. In some cases, the hazard is fixed immediately - like coiling up a hose to remove a tripping hazard. SORs are compiled and results used as leading indicators for workplace hazards. Additionally, each SOR input is tracked until the hazard risk is permanently reduced to acceptable levels.

7) Safety Assessment Reports (SARs): This process is a derivative of a behavior-based safety process where personnel are observed and at-risk behavior documented. There are several significant differences between the classic and the ROME versions, the most notable are 1) all observations are made voluntarily, i.e. no observations are made without the observed's permission and 2) the results of the process focuses on positive results of the observations. Anyone can take a few minutes to observe in-process work, not for work pace or oversight, but for safe work practices. Appropriate practices, such as using proper PPE and the correct tools, are noted as well as unsafe practices and potential use of best practices. Work interruption is minimal and the worker(s) receive quick, immediate feedback.

8) Safety Observation Predictive Metrics: Following the thought that an incident does not necessarily occur every time an unsafe act is performed, ROME uses several different inputs (first aid events, near misses and close calls, SORs, SARs, safety surveys, culture survey) to generate predictive metrics to identify unsafe acts, trend the overall behavior of the workforce, and use this information to raise the awareness of those risks. Additionally, the processes used to collect this data also are used to identify unsafe conditions. Often these conditions are identified by persons unfamiliar with the areas, and are taken for granted by the regular area workforce.

9) Safety Incentive Program: In FY 2007, ROME initiated a safety incentive program intended to increase employee involvement in the safety program. A third party was contracted to manage the program. The program did not meet the intent, and employee involvement remained low. Additionally, the predictive metrics derived from the program did not meld with the ROME metrics. In FY 2008, directorate-level incentive programs were implemented, and comparable funds were allocated. Besides not paying for the third-party administrative overhead costs, these 'locally owned and operated' programs significantly increased employee involvement. They utilize the existing predictive metric process tools and encourage participation through the participants becoming eligible for prize drawings. High quality of these employee inputs is encouraged through peer reviews, where significant findings and proactive behaviors are rewarded by cash awards.

10) Employee Safety Advisory Leadership Team (SALT): The SALT is an employee-run cross-functional safety committee that owns and operates the SAR program. The team generates reports on a monthly basis and shares the information with the ROME safety team, who meld the information into the safety observation predictive metrics. This program is one of ROME's most effective, with over 7,200 employee observations made in FY09.

11) Beyond Zero Focus Group (BZFG): This group is composed of members from all areas of ROME, and reports to the Beyond Zero Leadership Team (BZLT). The members have recommended multiple safety 
philosophy initiatives, such as linking the National Safety Council's (NSC) Weekly Safety Tip to several ROME-owned websites and sponsoring the NSC Family Safety \& Health magazine being sent to the homes of all ROME personnel. Most significantly, they developed a grass-roots presentation including a video which has been shown throughout Langley Research Center, including the Center director.

12) Beyond Zero Leadership Team: This team originated as the ROME Executive Safety Council, and is chaired by the ROME General Manager and facilitated by ROME Health, Safety, and Environmental Manager. Members include all ROME directors, representatives from all ROME safety committees, and labor union representatives. This team provides resources for safety initiatives, including those recommended by the BZFG, and helps ensure a common safety philosophy throughout ROME.

13) Subcontractor Safety Training: In 2005, ROME experienced several significant incidents which involved subcontractors. Investigation of these incidents revealed a common thread: subcontractors are expected to take risks, and they believed their behavior was the one desired. ROME began several initiatives to change that mindset, including training for all subcontractor personnel to ensure they understand the expectations of ROME and subcontractor owner/management seminars to assist their cultural shift from an 'acceptable risk' mentality to an 'everyone goes home unhurt' philosophy.

14) Management by Walking Around (MBWA): Management must lead and safety leadership requires visibility in the field and spending time with the persons who are at risk. This allows first-hand exposure to working conditions and to discuss safety (and other) issues with the workforce in their environment. Management is encouraged (indeed, measured) by their participation in the processes which require field observations.

\section{Hazard Identification, Work Process Reviews, and a Supportive Culture}

1) Perceived Expectations from Customers: A significant challenge to having a safe workforce is managing interactions with customer expectations. The desire to please the customer through meeting cost, schedule and quality expectations can drive a workforce to take undesired and unnecessary risks to meet those perceived expectations. The words and actions of the customer can reinforce those perceptions, despite their true intent and desire to perform safely. Discussions with key customers and their personnel explaining the impact of their words and actions can lead to a significant reinforcement of the zero-incident goal.

2) Safe-Plan-Of-Action and Safety Observation Reports: SPAs and SORs, previously noted, are both used to supplement formal planning inputs to the job such as procedures, work instructions, drawings, and risk assessments, providing a real-time, site-specific tool for the worker to assess any hazard assessment gaps from the formal tools. The hazard assessment gap can include a more "granular" look at a hazard, changing environmental conditions (i.e., weather, noise, lighting, tooling availability and condition, worker experience, more), hazards resulting from the physical work area (fall, impale, bump, ergonomics, more), and any other hazard identified by the work crew team.

3) Hazard recognition: An active industrial workplace is ever changing - tooling, hoses, raw material, shipping crates, and much more are used and moved continuously. With each of these moves, introduction of a hazard can occur. Other variables include tooling and equipment (especially portable) condition, individual worker proficiency at any particular activity, distractions (visitors, adjacent activities, lighting or noise changes, and more), time of day or week (worker thinking about other things, fatigue), and work urgency. Despite utilizing the hazard identification tools noted previously, this remains an area for improvement. ROME is increasingly emphasizing hazard recognition in various training regimens and is crucial to creating a robust worker positive active control as the final safety defensive "slice".

4) Manager to Coworker Responsibilities: OSHA states "Each employer...shall furnish to each of his employees a place of employment which is free from recognized hazards that are causing or are likely to cause death or serious physical harm to his employees" ${ }^{\text {"18 }}$. Management responsibilities go far beyond that: management needs to do whatever is necessary to ensure each and every employee has the tools, equipment, policies, and support needed for them to go home in as good (or better) condition than they arrived. This commitment is required from each ROME manager.

5) Ensuring Subcontractor Safety Performance Is Up to NASA/ROME Standards: A consistent weakness through early years of the ROME contract was subcontractor safety performance (Figure 3), measured by injuries, experienced or potential injury severity, and observed unsafe work practices (including some unsafe tooling/equipment). Despite contractual safety requirements, company culture of subs is often different than Jacobs and NASA - consider the unacceptable accident levels of industry averages. Over time, ROME management, in partnership with NASA, implemented the measures noted previously - relying not just on contractually obligated safety measures, but on active support, partnering, and increased oversight to ensure a safe workplace. Safety performance has significantly improved with more still to do. 
6) Learn From Others. It is prudent to learn from other's mistakes and Jacobs and ROME invest significant effort to understand causality of accidents in similar industries and apply lessons learned to local operations. Trade and safety magazines and journals often have information which can be used to avoid similar occurrences. Jacobs Technology utilizes a process where incidents are discussed weekly among members of management from the various groups, and lessons-learned are shared. At ROME, incidents which relate to workplace safety are documented in informative flyers

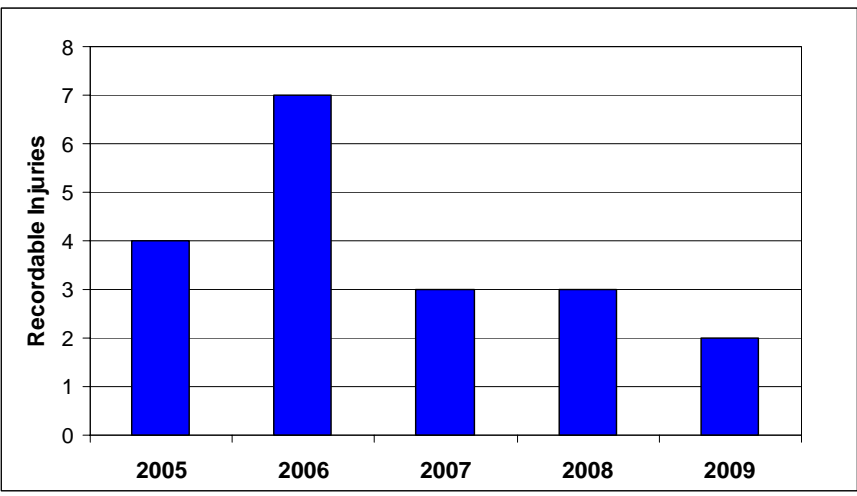

Figure 3. ROME Subcontractor Recordable Injury Rate and disseminated among the workforce.

And, as incidents can happen away from the workplace, these flyers often contain information which is useful at home as well.

7) Extensive provision of appropriate PPE and training: The use of PPE can seem like a "no-brainer", but past unsafe work practices at work and at home have embedded practices that exclude using PPE. The authors have even noted that in some cultures, it's not "cool” to use PPE. But PPE - safety glasses, hardhats, safety shoes, gloves, and other appropriate equipment - is often the last defense against getting hurt. Designed safety should never rely on PPE for primary protection, but as a backup for other measures. Jacobs and ROME provide all needed PPE for each employee at no cost to them. Work areas, sometimes based on specific work activity, and specific activities regardless of location are designated for required PPE use.

8) Extensive corporate support: A significant safety resource for ROME is being part of the larger JT and JE organizations. Corporate management grants significant autonomy to individual contract work sites, except for safety. During the entire process of proposing for and setting up a contract, safety is integrated through the process designs. During contract phase-in and early in a contract, corporate safety representatives are present and actively assist local safety professionals as well as functional managers. Through the life cycle of a contract, JT accomplishes (at least annually) Safety Evaluation Reviews, bringing managers from other contracts to inspect and score safety and environmental work sites and practices. This has the dual advantage of receiving improvement inputs from fresh eyes and also educating individual managers about what others are doing and about previously unrecognized hazards - a form of benchmarking best practices across the corporation. Additionally, within JT, every week senior management from every contract site and corporate participates in a safety telecom to review any/each injury and any/each motor vehicle accident. The goal is for all work segments to understand the cause of each incident, to learn from it, and to apply any resultant new measures as needed. Lastly, corporate resources are always made available if a particular safety issue requires it.

9) Stop work if it isn't safe: An important cultural and operational issue is that each worker must understand when it is acceptable to stop work due to a perceived safety hazard with unacceptably high risk. Jacobs and ROME policy is that safety will not be compromised and that work will be stopped to correct an unsafe condition. And there are different levels of stopping work (and associated impact) - locally and then up the system hierarchy. Stopping work will always have an impact on production. Of course, so will an accident. Additionally, the enthusiasm of the workers is typically pretty high to get the work done, so a challenge is ensuring each person knows that work can and will be stopped to address a safety issue. As noted earlier, ROME management at all levels must "walk the talk”, backing up workers that stop work and addressing safety concerns - even if the concern is determined to be unfounded. Experience has been that work stoppages are infrequent, but do occur, and annual work process surveys confirm that $98 \%$ of the workforce will stop work for an unsafe condition.

\section{A Culture of Caring}

Changing an organization's safety values from a one that accepts that some accidents are inevitable to one which will accept no less than incident-free, a personal and cultural philosophy shift must occur. Jacobs Technology and ROME have committed to creating and growing a culture that cares about the person - a total health approach that 
includes personal well-being at and away from work and includes the family. A series of educational and commitment workshops began in 2007 toward this cultural growth. This is not a program and is not intended to supplant or replace elements of the safety program. It does not override organizational responsibilities and authorities. People can't be treated like equipment and injuries are not just numbers. With this commitment goes responsibility; it's really a shifting from viewing safety as something required to working together for individual wellness. Much of the ROME workforce have committed to creating and nurturing a culture of caring, with the following as core values ${ }^{19}$ :

1) Value people for what they do and who they are: This is essentially a corollary of The Golden Rule. Managers often study methods and techniques to increase organizational performance and productivity, set new guidelines and rules, and monitor performance parameters. All of these are useful and necessary for an organization. Nowhere near as much time is spent on the organization's most important asset: people. Take the time to get to know people who work with and/or for you. You will probably find their issues and concerns, both on and away from the job, have similarities to yours.

2) Elevate safety to a personal value: Often time organizations will say safety is their number one priority. What does that really mean? All people perform tasks based on their perceived level of priority. If safety is just a priority in your organization, it is probable something can (and will) override it. Safety, whether realized consciously or not, is really a value, something which is a part of you and cannot be reprioritized.

3) Refuse to accept that "accidents happen": Most people discuss probability, not possibility. Most probability discussions are experienced-based, while possibility discussions help shape the future. Most people are comfortable with probability-based discussions, as they are safer and less controversial. Probability comes from the mind, possibility comes from the heart. If an incident-free workplace can be envisioned, incidents will never be seen the same again.

4) Make a personal commitment to create an environment free of accidents and injuries: This is more than just providing resources. It involves making tough decisions, ones which can affect customer satisfaction and future business opportunities. However, over the long term, customers will come to realize these decisions were not only to ensure no incidents occurred, but also had hidden value to them: cost, schedule, and quality commitments were not compromised by an incident.

5) Using accidents and near misses as opportunities to get better: Every incident has lessons to be learned from it. Incident analysis, if utilized properly, will nearly always point to a failure in management by allowing the conditions, cultures, and behaviors to exist which led to the incident. Many of these will require introspection and hard personal decisions to be made.

6) Rising from compliance mentality to a value mentality: Is OSHA compliance the appropriate level of safety? If so, why are there so many incidents in fully OSHA-compliant facilities? If personnel are valued and incidents avoided, compliance is not enough. Proper allocation of resources can demonstrate commitment to the workforce. Example: Worker needs to change a light bulb 18 feet above floor level. Normal operation calls for using a ladder. There is no OSHA issue with ladder use, but it isn't an optimal working condition. Improve the condition with a small manlift. This requires a small investment in equipment, but will reduce the potential for an incident and will increase worker efficiency while tangibly demonstrating a commitment to a safer workplace.

7) Courage: Once the commitment is made to develop a culture of caring, the challenges to staying the course will be considerable. In the beginning, internal and external customers will question the commitment. Initial results may not be promising. Consider the commitment a journey, one which will take the organization to higher levels of efficiency and customer satisfaction, and one which requires the courage to stay the course.

The ROME culture has changed as a result and it is still evolving. It is manifesting itself in many ways, both organized and spontaneous. A charity golf tournament was organized at the grass-roots level to provide a significant scholarship for the daughter of a friend and co-worker who suddenly passed away. ROME walks for health are held regularly. A grass-roots group formed, and the general manager backed with funding, to incentivize voluntary healthy activities such as weight-loss, aerobics training, getting a physical examination, and quitting smoking. At the personal level, individuals are taking more of a notice of a co-worker that doesn't seem to feel well, or seems to be down. Not intrusively, but caring. And it is understood throughout the management ranks that someone in need, work related or not, will be related to as a person first and an employee second. The culture change is real and has changed the way the authors interact and interchange with people at and away from work. 


\section{Results and Further Work}

As seen previously (Figure 1), ROME's safety performance, in terms of both recordable injuries and lost workdays, is significantly better than comparable organizations performing similar work (ROME vs. NAICS). However, in comparison with the various work segments across the whole of Jacobs Technology, ROME's recordable injury safety performance is substandard, placing ROME in the bottom third of all segments in recordable injury rate performance. The ROME team's efforts have exceeded the customer's expectations in terms of measurable metrics, but have not been able to consistently meet or exceed Jacobs Technology's performance goals. Severity levels of ROME injuries are comparable to other company organizations, but until injury rates are significantly reduced, the likelihood that a future injury will severely injure someone is unacceptably high. Performance feedback from the NASA customer makes clear that NASA shares the passion that striving for a zeroinjury workplace is clearly their goal as well.

ROME, as a team, is continuing to improve safety tools, work processes, and training to improve work quality and reduce workplace hazards. A specific step is to improve manager and supervisor engagement in the field. It is so easy to fall back on field time when workload and pressure to deliver is high. But engagement with the workforce is crucial to both understanding workplace issues and for providing leadership for integrating safety into all work practices - the people in the field are also busy and management must lead by example. The growth of employee-led safety and wellness functions and processes is exciting and will act as a catalyst for growing and strengthening the culture of caring. ROME will continue to grow people through training, targeted knowledge and career growth, and to utilize their inherent talents to better recognize the weak signals that indicate an accident may be getting ready to happen - then to take appropriate action to prevent its occurrence.

In the wind tunnel facilities, as staffing moves toward block operations (floating shifts of people working in more than one facility) with a blended workforce of contractor and civil servant personnel, it is imperative that a common culture of caring develop and joint use of safety tools be utilized. Thus, growth and improvement will be as a partnership of NASA and ROME people, many working physically together at tasks. It is clear to the authors that both ROME and NASA senior management are committed to working together to provide leadership to the workforce and that results will be shared.

In conclusion, Jacobs and ROME have brought much to NASA Langley Research Center resulting in improved safety and a growing culture of caring. Safety performance is still not good enough: the ROME team has not achieved the goal of zero injuries. ROME, in partnership with NASA, is continuing aggressive efforts to improve safety tools and training for hazard recognition while building a culture of caring. As this work continues, it is hoped that some of the tools, experiences, and the drivers for cultural change described in this paper can be utilized by others in industrial settings, resulting in zero injuries in their workplace.

\section{References}

${ }^{1}$ United States Department of Labor, Occupational Safety and Health Administration (2010), Voluntary protection programs: All about VPP, http://www.osha.gov/dcsp/vpp/all_about_vpp.

${ }^{2}$ United States Census Bureau, North American Industry Classification System, Code 5612 - Facilities Support Services, Recordable Injury Rate, 2008.

${ }^{3}$ United States Department of Labor, Bureau of Labor Statistics, Occupational Safety Table (10/29/2009), Incidence rates of nonfatal occupational injuries and illnesses by industry and case types, 2008, http://www.bls.gov/iif/oshwc/osh/os/ostb2071.pdf.

${ }^{4}$ National Council on Compensation Insurance: Safety Pays cooperative program with United States Department of Labor, Occupational Safety and Health Administration, http://www.osha.gov/dcsp/smallbusiness/safetypays/estimator.html.

${ }^{5}$ Virginia Workers' Compensation Commission (2010), http://www.vwc.state.va.us/bene_amts.htm.

${ }^{6}$ Reason, James (2000). Human Error: Models and Management. Business Management Journal. 320:768-770.

${ }^{7}$ ibid.

${ }^{8}$ Coutu, D. L. (2003). Sense and reliability: A conversation with celebrated psychologist Karl E. Weick. Harvard Business Review. 81(4), 84-90.

${ }^{9}$ Reason, J. (2004). Beyond the organisational accident: the need for "error wisdom" on the frontline. Quality \& Safety in Health Care. 13(2), ii28.

${ }^{10}$ Karoack, Mark A. (2009). Organizational Characteristics Associated With High Performance In Quality and Safety. http://mediasite.urmc.edu/mediasite/Viewer/Viewers/Viewer240TL3Banner.aspx?mode=Default\&peid=ab9d39ac-33fc-4237be9b-c50d97e54543\&pid=a7144c13-78d1-45ed-a5af-ae61043be426\&playerType=WM64Lite

${ }^{11}$ Taylor, M. (2005). More than fiscal trouble. Modern Healthcare. 35(24), 10.

${ }^{12}$ Zaharatos, Anthea (2002). An organization and employee-level investigation of the relationship between high-performance work systems and workplace safety. Queen's University at Kingston (Canada), Ph.D. dissertation. 
${ }^{13}$ United States Department of Labor, Occupational Safety and Health Administration (2010), Estimated costs of occupational injuries and illnesses and estimated impact on a company's profitability worksheet, http://www.osha.gov/dcsp/smallbusiness/safetypays/estimator.html [improve reference]

${ }^{14}$ Fitch, J. L., \& Fitch, B. E. (2004). Exploring Causative and Preventative Forces. Occupational Health \& Safety. 73(6), 119$125,+$.

${ }^{15}$ Leisten, D. (1997). The art and science of safety. World Wastes. 40(12), 52-55.

${ }^{16}$ Attrino, T. (1998). Risk managers finding it easier to justify sprinkler costs. National Underwriter (Property \& Casualty/Risk \& Benefits Management). 102(32), 3,16.

${ }^{17}$ Johnson, L. (1994). Preventing injuries: The big payoff. Personnel Journal. 73(4), 61-64.

${ }^{18}$ United States Department of Labor, Occupational Safety and Health Administration (2010), OSH Act of 1970, Section 5 Duties, http://www.osha.gov/pls/oshaweb/owadisp.show_document?p_table=OSHACT\&p_id=2743.

${ }^{19}$ Adapted over multiple revisions from original training material provided by JMJ Associates, Inc. 